\title{
Is homosexuality hard-wired?
}

A suggestion, based on measurements of post-mortem brains, that structures in the hypothalamus correlate with sexual behaviour, should be taken seriously. But the meaning is not yet clear.

THE empirical, illogical and unverifiable doctrine that 'every cloud has a silver lining' appears, against the odds, to apply even to the AIDS epidemic. At least, if it were not for the epidemic, Dr Simon LeVay from the Salk Institute at La Jolla, California, would not have been able to examine samples of brain tissue from 16 people known to have been male homosexuals nor to speculate on that basis (Science 253, 1034; 30 August 1991) that male sexual orientation is linked with the size of one of four particular nuclei (or stainably recognizable groups of neurons) in the hypothalamus.

LeVay's claim has naturally attracted a great deal of attention in newspapers around the world. The reasons are not far to seek, for one interpretation of his observations is that genetic males may behave homosexually because of variations in neural structures in the brain that may even be congenital. If that proves indeed to be the case, the tenor of much public argument about the morality of homosexuality would be transformed -- or should be. Could Christians, for example, continue to define homosexual behaviour as sinful if it were known to stem from some hard-wired neural structure?

Also naturally, the claim has attracted much scepticism. After all, the numbers are small, the work entails techniques unfamiliar outside neuroanatomy, the outcome of LeVay's study is genuinely surprising - and the implications could be considerable. But, of course, there is no reason why a result that is surprising should be scorned on that account alone, while those who know LeVay attest to the excellence of his work in neuroanatomy. (LeVay also volunteers that he is himself a homosexual.) So scepticism is inappropriate. Getting to grips with the significance of the observations is another matter.

It should be no surprise that there are neural structures that help to determine sexual behaviour. Distinctive neural structures are one obvious means by which gender-specific differences in sexual behaviour could be determined, and the natural place to look for them is in the hypothalamus, with its close links with the pituitary gland, which, with the gonads and to a lesser extent the adrenal glands, is the chief source of hormones regulating the reproductive system.

LeVay has been concerned with four particular nuclei in the hypothalamus (called INH1, 2, 3 and 4) which have previously been thought to be linked with gender-specific behaviour. He set out to tell whether, instead, they (or at least some of them) are determinants of sexual behaviour. Originally, he says, his ambition had been to compare the sizes of the four nuclei in male and female homosexuals, in each case matched with an appropriate control group. In the event, compiling a set of samples of brain tissue from female homosexuals proved to be impossible (for lesbian behaviour does not transmit human immunodeficiency virus or otherwise declare itself on death). So LeVay's study is based on three sets of brain tissue samples - one from a group of 19 declared male homosexuals; one from a group of 16 males, six of whom died of AIDS and ten of whom died of causes other than AIDS and whose sexual orientation is unknown; and one from a similar group of six women also "presumed heterosexual".

The presumption of heterosexuality in the two control groups sounds fishy, but does not weaken the study. LeVay is out to test the hypothesis that at least one of the four nuclei is, on the average, smaller in homosexual than in heterosexual males. If the 'presumed heterosexual' males included, by chance, some homosexuals, the result would simply be to decrease the significance of whatever difference is found in the comparison.

LeVay makes the technique sound much simpler than it can be. Take a section through the hypothalamus containing the four nuclei, slice than into 3 - micrometre sections, stain the nuclear cells and measure the geometrical area projected on each section and then calculate the volume of each nucleus by adding together the areas. The hypothalamus is bilaterally symmetrical, so that each tissue sample should yield two sets of measurements, and LeVay has made bilateral measurements on 70 per cent of his material.

The crucial comparison is between the 16 'presumed heterosexual' and the 19 known homosexual males. LeVay first concludes that there is no significant difference between the sizes of three of the nuclei (IHN1, 2 and 4). But there is, he says, a significant difference in the distribution of sizes measured for the third nucleus, IHN3, which thus becomes the presumed correlate of sexual orientation in male homosexuals. The claim is based on a one-sided analysis of variance - the sort of thing authors are driven to when referees will not accept a simple $t$ test.

Even so, there will be a great deal of argument about the data. One difficulty is that, in each group, the measured size of IHN3 spans a more than twentyfold range, from roughly 0.01 to $0.20 \mathrm{~mm}^{3}$. In other words, male homosexuality is not inconsistent with a large nucleus at the high end of the range, which in itself shows that the third nucleus is not an unambiguous determinant of sexual orientation. The size distributions from the homosexual and heterosexual groups differ chiefly in the clustering of about a third of the measurements around the smaller mean value.

On the assumption that the observations are confirmed (obviously important), what is to be made of LeVay's finding? First, there is the familiar difficulty of telling whether the observed difference is the cause of homosexual behaviour (in some) or the effect thereof. The scatter of the measured sizes suggests that nuclear size, if in any sense a 'cause', is neither a unique nor an unambiguous determinant of homosexual behaviour. Statements in some newspapers that LeVay "has found the seat of homosexuality" simply go too far, even if it is clear that the third nucleus (and probably the other three) are somehow involved with the determination of attributes of sexual behaviour.

There is also the possibility that both the measured differences of size and homosexual orientation are themselves the effects of some other cause. There are suggestive experiments in which it has been shown that male rats castrated at an early age develop female patterns of behaviour, and that females (not only rats) to whom testosterone is administered in early life will develop male behaviour. Plainly, the neural correlates of genetically determined gender are plastic at a sufficiently early stage.

In that light, it is almost certainly too soon to say that LeVay has shown that homosexuality is strictly genetically 'hardwired', although that may eventually prove to be the case. One distinguished practitioner says of LeVay's work that it puts the nail in the coffin of freudian attempts to account for homosexuality in terms of early interactions between parents and children. But even that is not necessarily true. Plastic structures in the hypothalamus allowing the consequences of early sexual arousal to be made permanent might suit the freudians well.

John Maddox 